Research Article

\title{
Blockade of Erythropoietin-Producing Human Hepatocellular Carcinoma Receptor B1 in Spinal Dorsal Horn Alleviates Visceral Pain in Rats
}

\author{
Chen-Li Sun $\mathbb{D},{ }^{1}$ Cheng-Wen Li $\mathbb{D},{ }^{2}$ Nong He $\mathbb{D},{ }^{2}$ Yuan-Zhang Tang $\mathbb{D},{ }^{1}$ Xiu-Liang Li $\left(\mathbb{D},{ }^{2}\right.$ \\ Fu-Shan Xue $\mathbb{D}^{2}{ }^{2}$ and Jia-Xiang $\mathrm{Ni} \mathbb{D}^{1}$ \\ ${ }^{1}$ Department of Pain Management, Xuanwu Hospital of Capital Medical University, Beijing, China \\ ${ }^{2}$ Department of Anesthesiology, Beijing Friendship Hospital, Capital Medical University, Beijing, China
}

Correspondence should be addressed to Fu-Shan Xue; xuefushan@aliyun.com and Jia-Xiang Ni; nijiaxiang@hotmail.com

Received 25 June 2020; Revised 19 February 2021; Accepted 10 March 2021; Published 7 April 2021

Academic Editor: Takahiro Ushida

Copyright (C) 2021 Chen-Li Sun et al. This is an open access article distributed under the Creative Commons Attribution License, which permits unrestricted use, distribution, and reproduction in any medium, provided the original work is properly cited.

\begin{abstract}
Objective. This experiment was designed to determine whether erythropoietin-producing human hepatocellular carcinoma (Eph) receptors were involved in the development of visceral pain. Methods. Adult male Sprague-Dawley rats were randomly divided into three groups receiving different treatments ( $n=16$ per group): intracolonic vehicle (control group), intracolonic 2, 4, 6trinitrobenzene sulfonic acid (TNBS) (TNBS group), and intracolonic TNBS and intrathecal EphB1 receptor blocking reagent (TNBS + EphB2-Fc group). Visceral hyperalgesia was evaluated with quantification of visceral pain threshold induced by colorectal distention. The spinal expressions of EphB1 and ephrinB2 and levels of their phosphorylated forms (p-EphB1 and p-ephrinB2) were assessed by Western blotting and immunohistochemistry. Results. The TNBS-treated rats developed significant visceral hyperalgesia. The spinal expressions of EphB1, p-EphB1, ephrinB2, and p-ephrinB2 were significantly increased in the TNBS group compared with the control group, but visceral hyperalgesia and elevation of spinal EphB1 and p-EphB1 expressions were evidently alleviated by intrathecal administration of EphB2-Fc in the TNBS + EphB2-Fc group. The number of EphB1- and p-EphB1-immunopositive cells, the average optical (AO) value of EphB1, and its phosphorylated form in the spinal dorsal horn were significantly increased in the TNBS group than in the control group, but they were obviously reduced by intrathecal administration of EphB2-Fc. There were no significant differences in the number of ephrinB2- and p-ephrinB2-immunopositive cells and the AO value of ephrinB2 and its phosphorylated form between the TNBS and TNBS + EphB2-Fc groups. Conclusion. EphB1 receptors in the spinal dorsal horn play a pivotal role in the development of visceral pain and may be considered as a potential target for the treatment of visceral pain.
\end{abstract}

\section{Introduction}

Abdominal pain is one of the major and troublesome hallmarks of gastrointestinal diseases, such as gastrointestinal tumors, inflammatory bowel disease, and irritable bowel syndrome. Visceral hyperalgesia, which refers to a decrease of visceral pain threshold to mechanical distension, is regarded as one of the pivotal players in the development of abdominal pain [1-3]. Increasing research has attempted to reveal the molecular mechanism of visceral hyperalgesia [4-8], but this issue is still not completely understood.
Erythropoietin-producing human hepatocellular carcinoma (Eph) receptors, including types $\mathrm{A}(\mathrm{A} 1-\mathrm{A} 10)$ and $\mathrm{B}$ (B1-B6), are the largest subfamily of transmembrane receptor tyrosine kinases (RTKs). It has been shown that the Eph receptors and their ligands Eph-receptor interacting proteins (ephrin) play a critical role in the development of the nervous system, such as regulation of axon guidance $[9,10]$. Currently, it has been known that ephrin ligands have two types: glycosylphosphatidylinositol (GPI) anchored type (ephrinA1-A10) and transmembrane type (ephrinB1-B3). Recently, accumulating evidence indicates that the ephrinB/EphB signaling pathway is a crucial player 
in the development of various chronic pains, including inflammatory pain, neuropathic pain, bone cancer pain, and viscerovisceral referred pain [11-20]. This signaling pathway can not only regulate the function of synapse [21-26] but also modulate chronic pain by interacting with $\mathrm{N}$-methyl-Daspartate (NMDA) receptors [20, 26-28] as well as other targets [29-31]. However, the importance of this signaling pathway is mainly identified in the development of somatic pain. Up to now, only a few studies are focused on the involvement of ephrinB2/EphB1 signaling pathway in the pathogenesis of visceral pain $[32,33]$. For example, a previous work using pelvic-urethra-related pain model found that ephrinB2 potentiated the pelvic-urethra reflex due to phosphorylation of EphB1 and/or 2 via the Src kinase [32]. Recently, another study using ephrinB2 knockout animals demonstrated that both ephrinB2 and EphB1 played important roles in 2, 4, 6-trinitrobenzene sulfonic acid- (TNBS) induced chronic (postinflammatory) visceral pain, but only ephrinB2 was involved in the development of stress-induced visceral pain [33]. Most important, it remains unclear whether administration of specific blockers to interrupt the phosphorylation of EphB1 and/or expression of ephrinB2 would attenuate visceral pain. Thus, this experiment was designed to assess the spinal expressions of EphB1 and ephrinB2 and their phosphorylation levels in a rat model of visceral pain induced by intracolonic injection of TNBS and determine the effects of using an EphB1 receptor blocking reagent on the development of visceral pain and the spinal expression of EphB1 receptors.

\section{Data and Methods}

2.1. Animals. After the experimental protocol was approved by the Animal Care and Use Committee of Xuanwu Hospital of Capital Medical University, adult male Sprague-Dawley (SD) rats, aged 8-10 weeks and weighing 150-250 g, were used in this experiment. Furthermore, all animal experiments were conducted in the Animal Experiment Center of Xuanwu Hospital. The animals were housed in plastic cages with soft bedding at room temperature and a 12:12-hour light-dark cycle every day. They were free to access food and water and acclimated for 3 days before the experiment. By using a randomized digital table, the rats were divided into three groups receiving different treatments $(n=16$ per group): intracolonic vehicle (control group), intracolonic TNBS (TNBS group), and intracolonic TNBS and intrathecal EphB1 receptor blocking reagent (TNBS + EphB2-Fc group).

2.2. Establishment of Colonic Hyperalgesia Model. In this experiment, a colonic hyperalgesia model was established by injection of TNBS (Sigma-Aldrich, MO, USA), as previously described [34, 35]. Briefly, the rats were anesthetized with $2 \%$ isoflurane and fasten in a supine position. A polyethylene catheter $(1.2 \mathrm{~mm}$ in diameter) was inserted into the colon, with the distal tip $6 \mathrm{~cm}$ far from the anus. The TNBS solution of $100 \mu \mathrm{l}(14-16 \mathrm{mg} / \mathrm{ml}$ in $25 \%$ ethanol) was injected into the colon via the catheter in the TNBS and TNBS + EphB2-Fc groups, and vehicle solution of $100 \mu \mathrm{l}$ including only $25 \%$ ethanol was injected in the control group. Then, the lower portion of the rat body was elevated for 30 seconds.

2.3. Histological Assessment of Colonic Damage. The level of colonic damage was evaluated before administration of TNBS (baseline), 1 day after administration of TNBS (inflammatory stage) and 15 days after administration of TNBS (postinflammatory stage). Four animals were euthanized under anesthesia with $2 \%$ isoflurane at each time-point. The $1 \mathrm{~cm}$ distal colon was excised, rinsed with saline, fixed in $4 \%$ formalin, and embedded in paraffin. Sections of fullthickness colon samples $(8 \mu \mathrm{m}$ thick) were stained with hematoxylin (Leica Biosystems) and eosin (Leica Biosystems) and observed under microscopy. The levels of colonic damage were scored according to the criteria described in previous literature [36, 37]: mucosal architecture loss (0-3); goblet cell depletion ( 0 , absent; 1 , present); crypt abscess ( 0 , absent; 1 , present); cellular infiltration (0-3); and tunica muscularis thickening (0-3).

2.4. EphB2-Fc Preparation and Intrathecal Injection. This experiment used mouse recombinant chimaera of EphB2-Fc (Bio-Techne, MN, USA) as an EphB1 receptor blocking reagent, as reported in previous studies with rats $[17,18,24]$. By combining with the endogenous ephrinB, EphB2-Fc can cause EphB1 substituted and cleaved, and then result in the blockade of the downstream signals of EphB1. EphB2-Fc chimaera was prepared on the day of intrathecal injection $(100 \mu \mathrm{g} / \mathrm{ml}$ in sterile PBS). On 14, 15, and 16 days after intracolonic injection of TNBS or vehicle, the rat was anesthetized with $2 \%$ isoflurane and lumbar puncture was performed at L4-5 intervertebral space. When successful intrathecal puncture was confirmed by a typical tail-flick, EphB2-Fc of $5 \mu \mathrm{l}$ was administrated in the TNBS + EphB2-Fc group and vehicle (sterile PBS) of $5 \mu \mathrm{l}$ in the other groups, once a day for three consecutive days.

2.5. Behavioral Test. In each animal, colorectal distension (CRD) was carried out 17 days after intracolonic injection of TNBS or vehicle, as previously described $[4,33,38]$. In brief, a latex double-lumen catheter was attached to a balloon dilator with a diameter of $5 \mathrm{~mm}$. The lubricated dilator was gently inserted into the descending colon until its distal tip was $6 \mathrm{~cm}$ from the anus. Then, CRD was maintained by injection of increasing air $(0.1-5.0 \mathrm{ml})$. The rats were placed in a small lucite cubicle in which they were kept waking up and acclimated for $30 \mathrm{~min}$ before CRD.

The abdominal withdrawal reflex (AWR) responses, which referred to a sudden and persistent abdominal muscle contraction with abdomen lift off the platform, were evaluated by an observer who was blind to the group assignment. The volume of injected air that elicited an observable AWR was used to quantify the visceral pain threshold. For each rat, the behavioral test was performed three times with an interval of $5 \mathrm{~min}$. The averaged value of three measurements was used as the visceral pain threshold. 
2.6. Western Blotting. After the behavioral test, animals were euthanized under anesthesia with $2 \%$ isoflurane, and then the spinal cord at lumbosacral levels (L6-S1) was freshly extracted and stored in liquid nitrogen. To quantify the expression levels of EphB1, ephrinB2, and their phosphorylated forms (p-EphB1 and p-ephrinB2) in the spinal cord by Western blotting, spinal samples were homogenized in ice-cold $\left(4^{\circ} \mathrm{C}\right)$ lysis buffer containing $50 \mathrm{mM}$ Tris- $\mathrm{HCl}, 150 \mathrm{mM} \mathrm{NaCl}, 1 \%$ Triton X-100, 0.5\% deoxycholate, $0.1 \%$ SDS, $0.2 \mathrm{mM}$ EDTA, $10 \mathrm{mM} \mathrm{NaF}, 10 \mu \mathrm{g} / \mathrm{ml}$ aprotinin, $1 \mu \mathrm{g} / \mathrm{ml}$ leupeptin, $10 \mu \mathrm{g} / \mathrm{ml}$ pepstatin, $0.4 \mathrm{mM}$ 4-(2-amino-ethyl)-benzenesulfonyl fluoride, and $1 \mathrm{mM}$ sodium orthovanadate ( $\mathrm{pH} 7.5)$. Next, precipitation procedures were conducted on the homogenate. The protein concentrations were analyzed using a BCA protein assay kit (Pierce Biotechnology, MA, USA). These proteins were separated with $8 \%$ or $10 \%$ SDS-PAGE and then transferred to a nitrocellulose membrane.

The protein levels were detected by antibodies including anti-EphB1 (Santa Cruz Technology, CA, USA), anti-phosphorylated EphB1 (Santa), anti-ephrinB2 (Bioss Biotechnology, MA, USA), and anti-phosphorylated ephrinB2 (Bioss) antibodies. The membrane was blocked with $5 \%$ milk in Trisbuffered saline for $1 \mathrm{~h}$, incubated with anti-phosphorylated EphB1 $(1: 1000)$ and anti-phosphorylated ephrinB2 $(1: 1000)$ antibodies, and then incubated with horseradish peroxidaseconjugated secondary antibody. The bands were processed by enhanced chemiluminescence reagents (Millipore, MA, USA). The same membrane was stripped and processed with primary antibodies against EphB1 $(1: 1000)$ and ephrinB2 $(1: 1000)$.

The films were digitized, and densitometric quantification of immunoreactive bands was performed by using the Image $J 1.51$ software (Softonic International, Barcelona, Spain). The expression of a specific protein was normalized to that of $\beta$-tubulin.

2.7. Immunohistochemistry. The lumbosacral spinal cord sample was fixed in $4 \%$ formalin and embedded in paraffin. Sections $8 \mu \mathrm{m}$ thick were incubated with polyclonal rabbit antibodies of anti-EphB1 (1:200, Santa), anti-ephrinB2 (1: 200, Santa), anti-phosphorylated EphB1 (1:200, Santa), and anti-phosphorylated ephrinB2 $(1: 200$, Santa). The antirabbit IgG antibody ( $1: 200$, Sigma) was used to incubate the tissue sections. After the tissue slides were incubated with ABC reagent ( $1: 200$, Thermo Fisher Scientific), they were strained with diaminobenzidine solution for 1 to $2 \mathrm{~min}$ and then rinsed in distilled $\mathrm{H}_{2} \mathrm{O}_{2}$. Next, the tissue slides were strained with hematoxylin solution for 1 to $2 \mathrm{~min}$ and were then rinsed in distilled $\mathrm{H}_{2} \mathrm{O}_{2}$ again. Normal goat serum was used as a negative background control. Finally, EphB1 and ephrinB2 immunopositive cells in the spinal dorsal horn were counted and the integrated optical density values were measured by the IPP 6.0 software (Media Cybernetics). Averaged optical $(\mathrm{AO})$ values were calculated by the ratio of integrated optical density/area of tissue.

2.8. Statistical Analysis. All data are expressed as the mean \pm standard error of mean (SEM). The differences in the spinal expression levels of detected proteins and the threshold of visceral pain among groups were analyzed using one-way analysis of variance (ANOVA) with the SPSS software (Version 20.0, International Business Machines Corporation, NY, USA). A $P$ value of less than 0.05 was considered statistically significant.

\section{Results}

3.1. Colonic Damage. As shown in Figure 1, microscopic scores of colonic damage were markedly increased at 1 day after administration of TNBS compared with those at the baseline. However, microscopic scores of colonic damage were significantly decreased at 15 days after administration of TNBS compared with those at 1 day after administration of TNBS. Furthermore, there was no difference in the microscopic scores of colonic damage between the baseline and 15 days after administration of TNBS.

3.2. Behavioral Test. As shown in Figure 2, the visceral pain threshold was significantly decreased in the TNBS group compared with the control group. Intrathecal administration of EphB2-Fc evidently attenuated the TNBS-induced visceral hyperalgesia.

3.3. Spinal Expressions of EhpB1, ephrinB2, p-EphB1, and p-ephrinB2. As compared with the control group, spinal expression levels of EphB1 and p-EphB1 were markedly elevated in the TNBS group, and these expression evaluations were significantly decreased by intrathecal administration of EphB2-Fc in the TNBS +EphB2-Fc group (Figure 3(a)).

As compared with the control group, the spinal expression level of ephrinB2 was significantly increased in the TNBS group, with a remarkable upregulation of p-ephrinB2 expression. However, intrathecal administration of EphB2Fc failed to suppress the TNBS-induced expression upregulation of two proteins (Figure 3(b)).

3.4. Immunohistochemical Assay for EhpB1, ephrinB2, p-EphB1, and p-ephrinB2 in the Spinal Dorsal Horn. When goat serum was used instead of the first antibody for a negative background control, no immunopositive cell was noted (Figure 4(a)). The representative immunohistochemical staining images of EphB1, ephrinB2, and their phosphorylated forms distribution in the spinal dorsal horn are shown in Figure 4(b). The expression levels of these proteins were obviously increased in the TNBS group compared to the control group. The expression levels of EphB1 and p-EphB1 were markedly reduced in the TNBS + EphB2-Fc group compared to the TNBS group, but expression levels of ephrinB2 and p-ephrinB2 were not different between TNBS and TNBS + EphB2-Fc groups.

The numbers of EphB1- and p-EphB1-immunopositive cells in the spinal dorsal horn were significantly increased in the TNBS group compared to the control group, but they were obviously decreased by intrathecal administration of EphB2-Fc. The numbers of ephrinB2- and p-ephrinB2- 


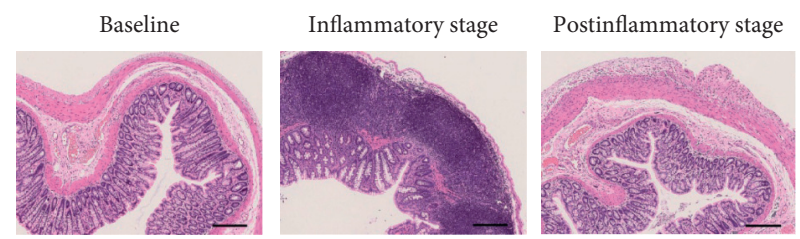

(a)

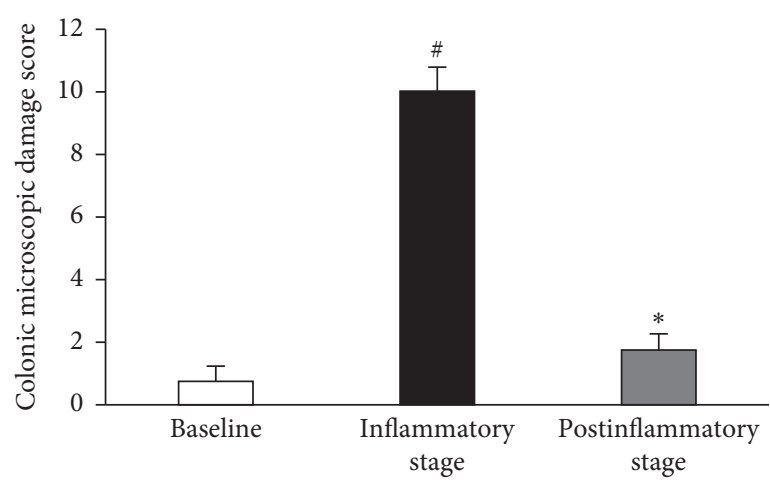

(b)

FIGURE 1: (a): The typical photomicrographs of colonic HE staining before administration of TNBS (baseline), 1 day after administration of TNBS (inflammatory stage), and 15 days after administration of TNBS (postinflammatory stage); (b): comparisons of colonic microscopic damage scores among three time-points. $n=4$ in each group. Data are expressed as the mean \pm SEM. ${ }^{\#} P<0.05$ versus Baseline. ${ }^{*} P<0.05$ versus inflammatory stage. Scale bar $=250 \mu \mathrm{m}$.

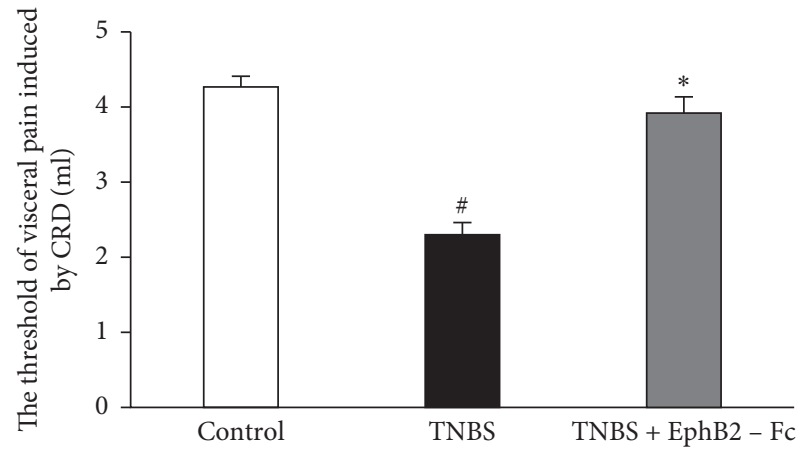

Figure 2: The comparisons of visceral pain thresholds induced by the colorectal distension among three groups. $(n)=8$ in each group. Data are expressed as the mean \pm SEM. ${ }^{\#} P<0.05$ versus the control group. ${ }^{*} P<0.05$ versus TNBS group.

immunopositive cells were significantly increased in the TNBS group compared with the control group. There was no significant difference in the numbers of ephrinB2- and p-ephrinB2-immunopositive cells between TNBS and TNBS + EphB2-Fc groups (Figure 5)

The AO values of EphB1, ephrinB2, and their phosphorylated forms in the spinal dorsal horn are shown in Figure 6. As compared to the control group, AO values of EphB1 and p-EphB1 were obviously elevated in the TNBS group. Intrathecal administration of EphB2-Fc significantly suppressed the TNBS-induced increase of $\mathrm{AO}$ values of EphB1 and p-EphB1. The AO values of ephrinB2 and p-ephrinB2 were obviously higher in the TNBS group than in the control group, but they were not decreased by intrathecal administration of EphB2-Fc.

\section{Discussions}

The present experiment aimed to assess the effects of EphB1 receptor blockade on the development of visceral hyperalgesia and spinal expressions of EphB1 and ephrinB2 in a rat model of visceral pain induced by TNBS. Our results showed that EphB1 receptor blockade with EphB2-Fc could significantly alleviate visceral hyperalgesia and decrease spinal expression of EphB1.

Accumulating evidence indicates that ephrinB/EphB signaling is a critical player in the development of somatic and neuropathic pain. The expression of EphB1 is upregulated and the nociceptive behaviors of thermal hyperalgesia and/or mechanical allodynia are induced by applying cutaneous inflammation [27], peripheral nerve injury $[11,14,16,24]$, or carcinoma cells inoculation $[17,18,39]$. In the neuropathic pain models evoked by chronic constriction nerve injury [12-14] and/or partial nerve ligation [13], however, these nociceptive behaviors can be alleviated by interrupting the EphB1 signaling pathway with EphB1 small interfering RNA [14] or knockout technique [12, 13]. Furthermore, blockade of EphB1 receptors has been demonstrated to produce a remarked alleviation on these nociceptive behaviors in the rodents evoked by chronic constriction nerve injury $[12,24,29,30]$, tumor cell implantation $[18,39]$, or subcutaneous remifentanil infusion $[27,31]$. By using a visceral hyperalgesia model induced by intracolonic TNBS, this experiment showed that intrathecal administration of EphB1 receptor blocking reagent significantly alleviated visceral hyperalgesia with downregulation of spinal EphB1 expression. This is in accord with the results of a recent study [33], in which visceral hyperalgesia induced by intracolonic TNBS in wild-type mice did not occur in the ephrinB2 knockout mice.

Our results also indicated that intracolonic TNBS-provoked visceral hyperalgesia, with a significant upregulation of spinal p-EphB1 expression. The EphB1 receptor blocking reagent not only alleviated the upregulation of spinal EphB1 expression but also decreased the level of spinal p-EphB1 in the rats with TNBS-induced visceral pain. This suggested that phosphorylation of EphB1 receptors may play a critical role in the development of postinflammatory visceral hyperalgesia. Even previous studies demonstrated that EphB1 receptor agonist could induce transient thermal hyperalgesia in the rats $[18,24,28,29]$, with an increased phosphorylation level of NR2B (a subunit of NMDA 


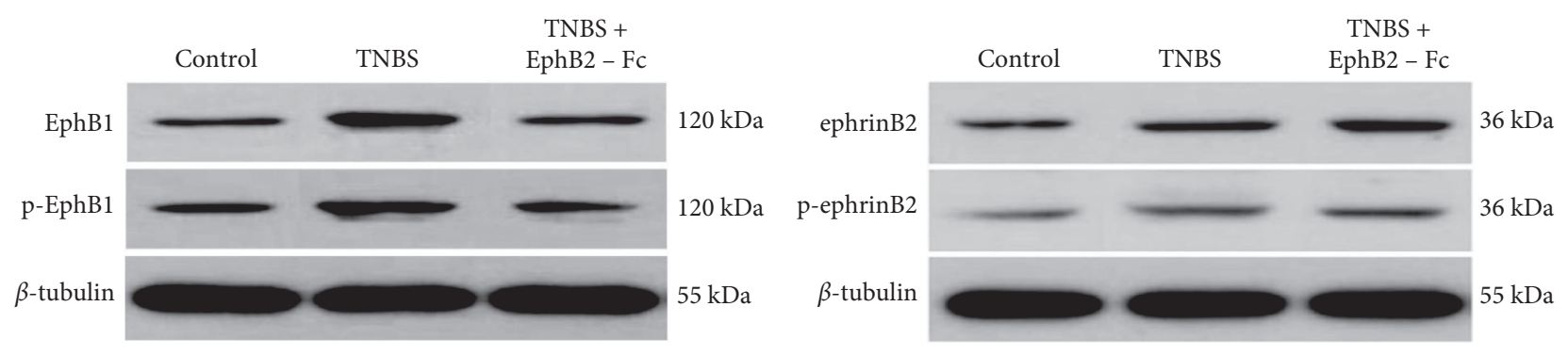

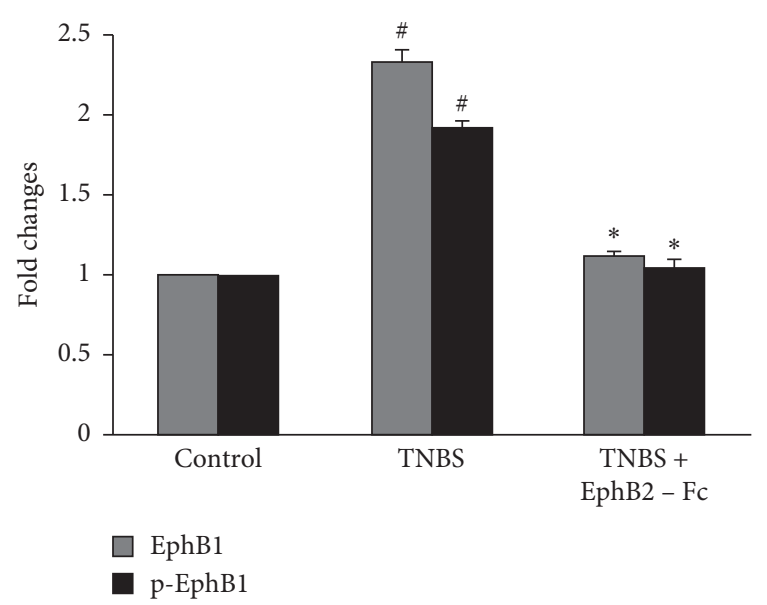

(a)

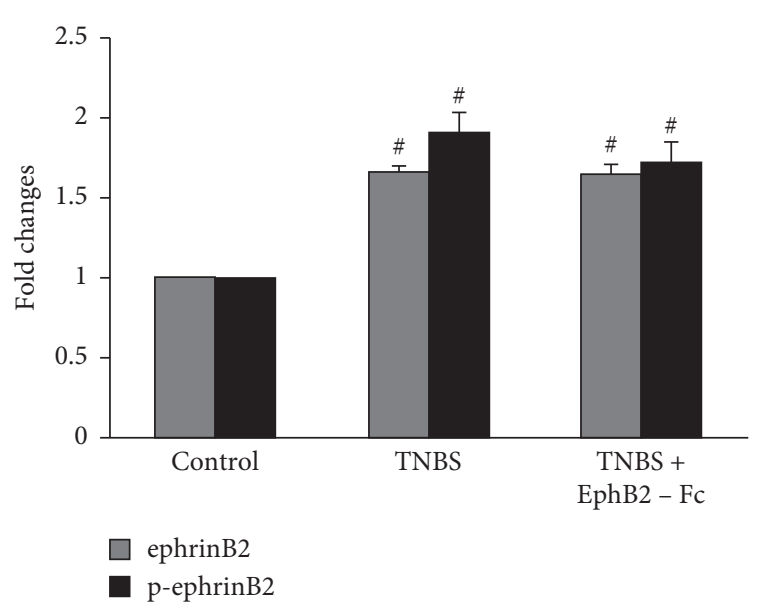

(b)

FIgURE 3: The expression levels of spinal EphB1 and p-EphB1 (a), and ephrinB2 and p-ephrinB2. (b) The upper parts of pictures are the representative Western blot bands and the lower parts are comparisons of their expression levels among three groups. $(n)=8$ in each group. Data are expressed as the mean \pm SEM. ${ }^{\#} P<0.05$ versus control group. ${ }^{*} P<0.05$ versus TNBS group.
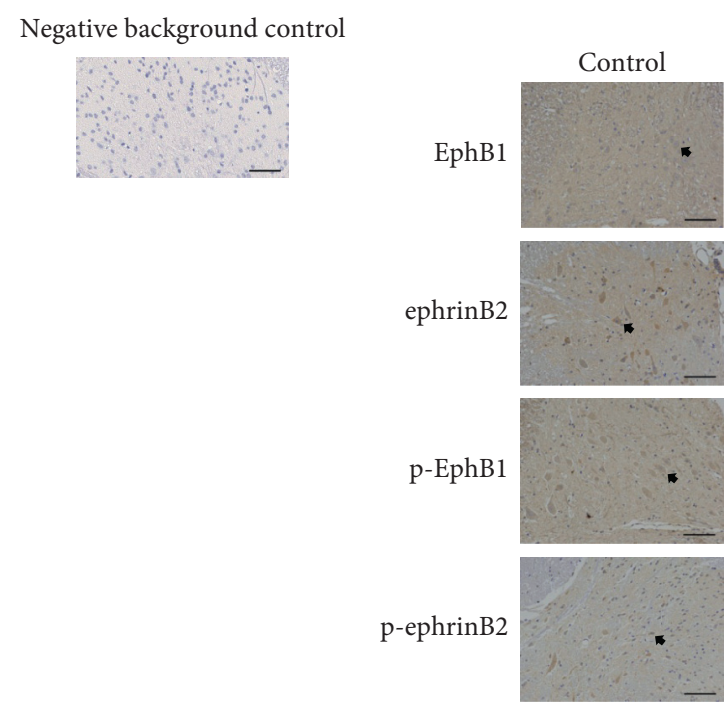

(a)

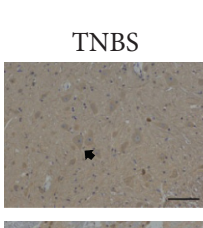

TNBS + EphB2 - Fc
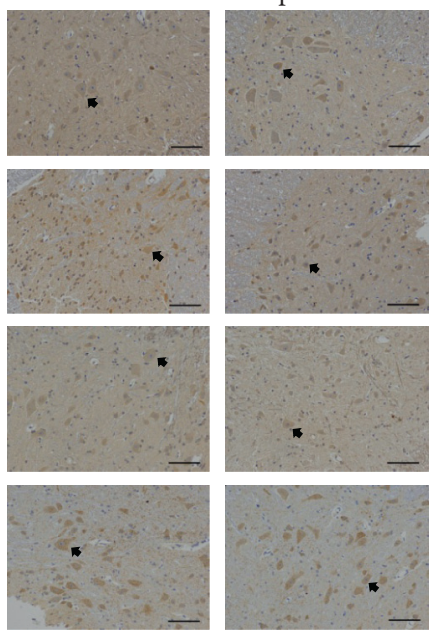

(b)

FIGURE 4: (a): The typical photomicrographs of immunostaining for negative background control; (B) EphB1, ephrinB2, and their phosphorylated forms in the spinal dorsal horns of three groups (b). Arrows indicate representative EphB1-, ephrinB2-, p-EphB1-, or pephrinB2-immunopositive cells. Scale bar $=50 \mu \mathrm{m}$.

receptors) in the spinal cord [18, 28], which was reversed via pretreatment of Src-family kinase inhibitor [28]. This transient thermal hyperalgesia was not related to the upregulation of total EphB1 but was dependent on the activation of EphB1, indicated by the upregulation of phosphorylated NR1 (a subunit of NMDA receptors) and 


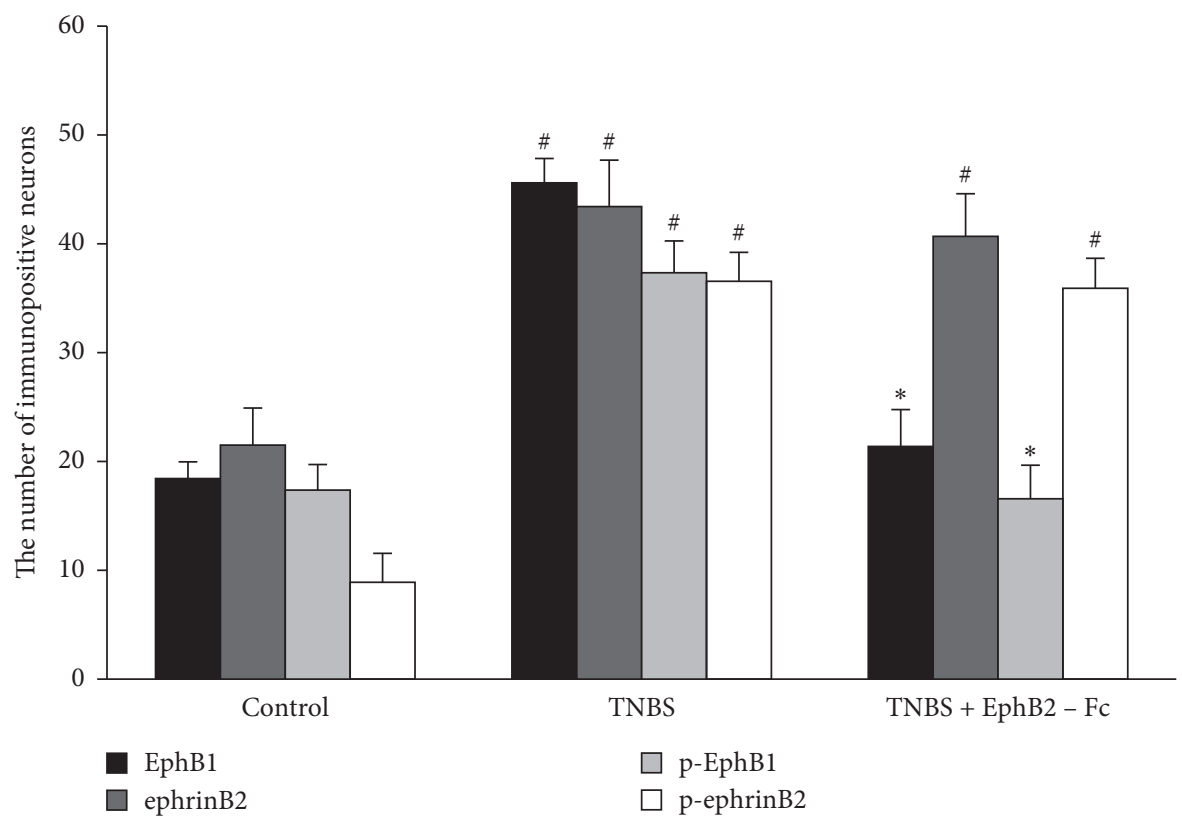

Figure 5: The comparisons for the numbers of EphB1-, ephrinB2-, p-EphB1-, and p-ephrinB2-immunopositive cells in the spinal dorsal horn among three groups. $(n)=4$ in each group. Data are expressed as the mean \pm SEM. ${ }^{\#} P<0.05$ versus the control group. ${ }^{*} P<0.05$ versus the TNBS group.

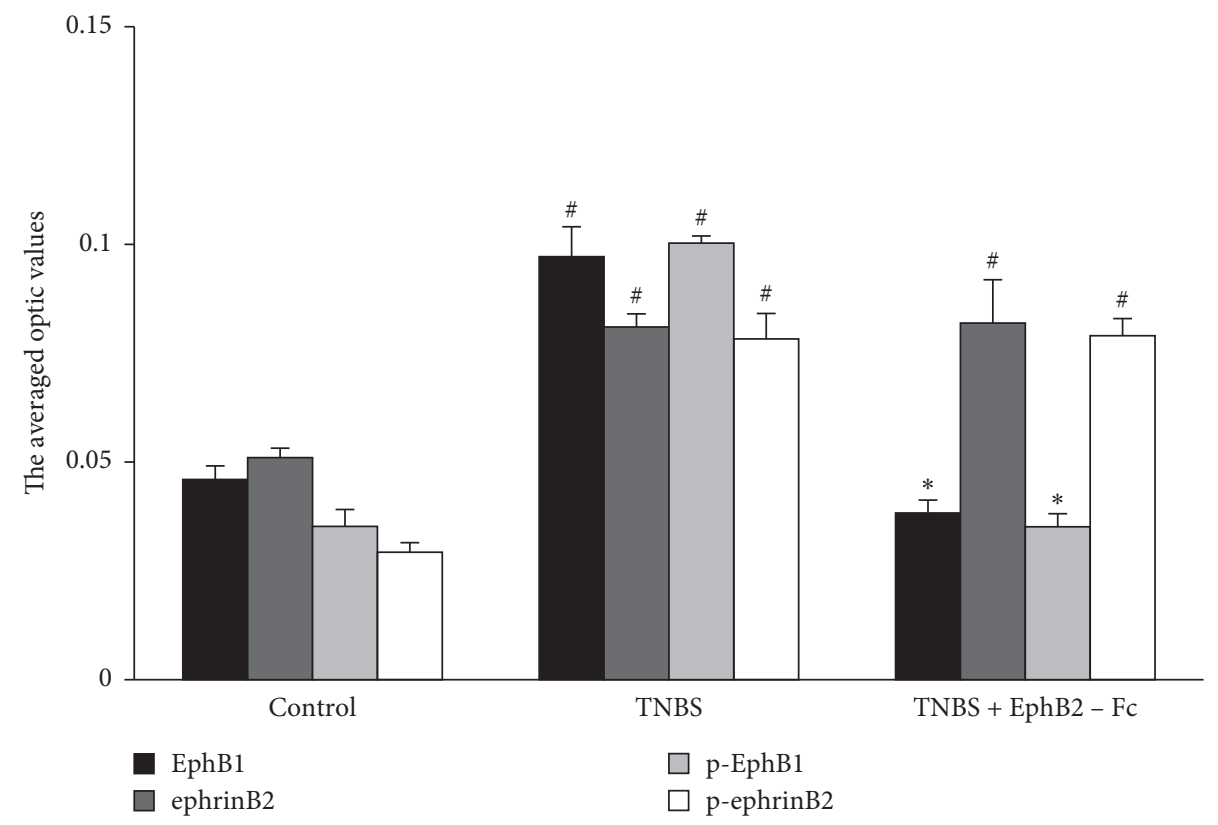

FiguRE 6: The comparisons for the averaged optic values of EphB1, ephrinB2, and their phosphorylated forms in the spinal dorsal horn among three groups. $n=4$ in each group. Data are expressed as the mean \pm SEM. ${ }^{\#} P<0.05$ versus the control group. ${ }^{*} P<0.05$ versus the TNBS group.

NR2B [18]. Additionally, the pretreatment with NMDA receptor antagonist could eliminate thermal hyperalgesia and mechanical allodynia in the rats receiving an intrathecal administration of EphB1 receptor agonist (ephrinB1-Fc) [27]. Similarly, knockdown of ERK5 inhibited activation of cAMP-responsive element-binding protein (CREB) and alleviated thermal hyperalgesia and mechanical allodynia provoked by intrathecal administration of EphB1 receptor agonist (ephrinB2-Fc) [29]. These findings of previous studies demonstrate that NMDA receptors, Src-family kinase, and ERK/CREB may be the potential downstream signal transducers of EphB1 receptors, and the interactions between EphB1 receptor and its downstream signal transducers may be crucial for the development of hyperalgesia and allodynia induced by intrathecal administration of EphB1 receptor agonist. The results of the present 
experiment were similar to the findings from a previous study by Slack and colleagues [28], in which the pretreatment with EphB1 receptor blocking reagent attenuated thermal hyperalgesia and mechanical allodynia and inhibited the upregulation of phosphorylated NR2B expression in an inflammatory pain model induced by intraplantar injection of carrageenan. Furthermore, Peng and colleagues [20] reported that the pretreatment with Src inhibitor could reverse phosphorylation of Src and NR2B receptors and alleviate the urethra reflex sensitization in the rats of mustard oil-induced acute colitis. The same results have been also described in a previous study with the pretreatment using a NMDA receptor antagonist in the rats receiving intrathecal administration of ephrinB2 [32]. The pretreatment with EphB receptor block reagent can not only produce these effects achieved by the pretreatment with Src inhibitor or NMDA receptor antagonist but also reverse the phosphorylation of EphB1/2 [20, 32]. In addition, the pretreatment with a NMDA receptor antagonist has been demonstrated to eliminate thermal hyperalgesia and mechanical allodynia in the rats receiving intraplantar injection of remifentanil [27]. These previous findings suggest that both NMDA receptors and Src-family kinase are likely to be downstream signal transducers of EphB1 receptors, through which EphB1 receptors mediate in inflammatory pain and viscerovisceral referred pain.

As described by Klein [40], ephrin-Eph system can function in a bidirectional fashion, to trigger reverse signaling into the ephrin-expressing cells, forward signaling into the Eph-expressing cells, or bidirectional signaling into both cells; that is, Eph receptors can act as a ligand and an ephrin ligand can also play a receptor role. However, this experiment showed that EphB1 blocking reagent failed to reduce the upregulation of spinal ephrinB2 and p-ephrinB2 expressions in the TNBS-treated rats. This indicates that EphB1 blocking reagent maybe plays a forward role by inhibiting activation of related downstream receptors and kinases. Previous studies in the bone cancer pain model and CCI-induced neuropathic pain model demonstrated that EphB1 receptor antagonist relieved hyperalgesia and allodynia by downregulating phosphorylation of NR1, NR2B, Src, ERK, MK2, and CREB or reducing activation of ERK5/ CREB in the spinal cord $[18,29]$. Thus, similar signaling mechanisms may also be attributable to postinflammatory visceral hyperalgesia induced by TNBS in this experiment. In fact, EphB1 receptors have been identified in the spinal cord, locating in the neurons [11, 16-18], astrocytes, and microglial cells [17]. Thus, the detailed role of phosphorylation of spinal EphB1 receptors in the development of visceral pain warrants future study.

As a relay station for pain signals, the spinal dorsal horn has many signal transducers involving pain. In the bone cancer pain model induced by tumor cell implantation $[17,39]$ and the neuropathic pain model provoked by chronic constriction nerve injury [11] or crush spinal nerve injury [16], the immunostaining and/or immunofluorescence assessment shows that EphB1 receptors are mainly distributed in the spinal dorsal horn. In the rats with tumor cell implantation-evoked bone cancer pain by immunoblotting [39], moreover, ephrinB2 ligands have been observed to be mainly located in the spinal dorsal horn. In this experiment, thus, the numbers of EphB1, ephrinB2, p-EphB1, and p-ephrinB2 immunopositive cells in the dorsal horn and their $\mathrm{AO}$ values were assessed by immunohistochemistry to further determine the changes of their expressions in the spinal dorsal horn. Similar to our results obtained by the Western blotting, intrathecal administration of EphB1 receptor blocking reagent significantly attenuated visceral hyperalgesia, with a significant downregulation of EphB1 and p-EphB1 expressions in the spinal dorsal horn in the TNBS-treated rats. However, intrathecal administration of EphB1 blocking reagent failed to decrease the upregulation of ephrinB2 and p-ephrinB2 expressions in the spinal dorsal horn in the TNBS-treated rats. These results further support that the EphB1 receptors in the spinal dorsal horn play a pivotal role in the development of TNBS-induced visceral pain.

It must be pointed out that there are several limitations in the design of this experiment. First, as intrathecal administration of EphB1 receptor blocking reagent has shown an inability to change normal pain perception in the rats [18], a group of animals receiving EphB1 receptor blocking reagent on 14,15, and 16 days after the intracolonic vehicle was not included. Second, as the behavioral test was not performed before the initial intervention, it was unable to compare the visceral pain thresholds before and after intervention. Third, this experiment was mainly focused on the role of the ephrinB2-EphB1 signaling pathway in the development of visceral pain. It cannot provide any clue regarding the possible contributions of the related downstream signaling pathways to the development of visceral hyperalgesia. Thus, further experiments are still needed to address these issues.

In conclusion, this experiment demonstrates that blockade of spinal EphB1 receptors is able to alleviate the visceral hyperalgesia and reduce upregulation of spinal EphB1 expression in a rat model of TNBS-provoked visceral pain. It indicates that spinal EphB1 receptors play a crucial role in the development of visceral pain and may be a promising target of visceral pain treatment.

\section{Abbreviations}

Eph: Erythropoietin-producing human hepatocellular carcinoma

RTKs: Receptor tyrosine kinases

EPHRIN: Eph-receptor interacting proteins

GPI: Glycosylphosphatidylinositol

NMDA: N-Methyl-D-aspartate

TNBS: 2, 4, 6-Trinitrobenzene sulfonic acid

SD: $\quad$ Sprague-Dawley

HE: Hematoxylin-eosin

CRD: Colorectal distension

AWR: Abdominal withdrawal reflex

AO: $\quad$ Averaged optical

SEM: $\quad$ Standard error of the mean

ANOVA: Analysis of variance

CREB: CAMP responsive element-binding protein. 


\section{Data Availability}

The data are available on request.

\section{Disclosure}

Fu-Shan Xue and Jia-Xiang $\mathrm{Ni}$ are the correspondence authors.

\section{Conflicts of Interest}

The authors declare that they have no conflicts of interest.

\section{Authors' Contributions}

Chen-Li Sun considerably contributed to the design and implementation of the experiment, data collection, analysis, and interpretation and drafted the manuscript. Fu-Shan Xue and Jia-Xiang Ni equally contributed to the conception and design of the experiment and fund acquisition, helped to draft the manuscript and critically revised it. Cheng-Wen Li, Nong He, Yuan-Zhang Tang, and Xiu-Liang Li participated substantially in the implementation of the experiment, data acquisition, and interpretation. All authors had read and approved the final manuscript.

\section{Acknowledgments}

This study was supported by the Basic and Clinical Research Collaboration Foundation of Capital Medical University (no. 303-01-007-0169) and Beijing Municipal Administration of Hospitals Youth Program (no. 008-0113).

\section{References}

[1] M. Kanazawa, M. Hongo, and S. Fukudo, "Visceral hypersensitivity in irritable bowel syndrome," Journal of Gastroenterology and Hepatology, vol. 26, no. 3, pp. 119-121, 2011.

[2] W. L. Hasler and C. Owyang, "Challenges of managing pain in constipation-predominant IBS: clinical perspectives on antinociceptive actions of linaclotide," Gastroenterology, vol. 145, no. 6, pp. 1196-1199, 2013.

[3] D. A. Drossman, "Functional gastrointestinal disorders: history, pathophysiology, clinical features and rome IV," Gastroenterology, vol. 16, 2016.

[4] Y. Lu, B.-C. Jiang, D.-L. Cao, L.-X. Zhao, and Y.-L. Zhang, "Chemokine CCL8 and its receptor CCR5 in the spinal cord are involved in visceral pain induced by experimental colitis in mice," Brain Research Bulletin, vol. 135, pp. 170-178, 2017.

[5] Y. Liu, W. Liu, X. Wang, Z. Wan, Y. Liu, and Y. Leng, "Dexmedetomidine relieves acute inflammatory visceral pain in rats through the ERK pathway, toll-like receptor signaling, and TRPV1 channel," Journal of Molecular Neuroscience, vol. 66, no. 2, pp. 279-290, 2018.

[6] F. Fan, Y. Tang, H. Dai et al., "Blockade of BDNF signalling attenuates chronic visceral hypersensitivity in an IBS-like rat model," European Journal of Pain, vol. 24, no. 4, pp. 839-850, 2020.

[7] C. Cruz and F. Cruz, "The ERK 1 and 2 pathway in the nervous system: from basic aspects to possible clinical applications in pain and visceral dysfunction," Current Neuropharmacology, vol. 5, no. 4, pp. 244-252, 2007.
[8] L. Y. Qiao and M. A. Gulick, "Region-specific changes in the phosphorylation of ERK12 and ERK5 in rat micturition pathways following cyclophosphamide-induced cystitis," American Journal of Physiology-Regulatory, Integrative and Comparative Physiology, vol. 292, no. 3, pp. 1368-1375, 2007.

[9] D. G. Wilkinson, "Multiple roles of EPH receptors and ephrins in neural development," Nature Reviews Neuroscience, vol. 2, no. 3, pp. 155-164, 2001.

[10] Y. Goldshmit, S. McLenachan, and A. Turnley, "Roles of Eph receptors and ephrins in the normal and damaged adult CNS," Brain Research Reviews, vol. 52, no. 2, pp. 327-345, 2006.

[11] X.-J. Song, J.-L. Cao, H.-C. Li, J.-H. Zheng, X.-S. Song, and L.-Z. Xiong, "Upregulation and redistribution of ephrinB and EphB receptor in dorsal root ganglion and spinal dorsal horn neurons after peripheral nerve injury and dorsal rhizotomy," European Journal of Pain, vol. 12, no. 8, pp. 1031-1039, 2008.

[12] Y. Han, X. S. Song, W. T. Liu, M. Henkemeyer, and X. J. Song, "Targeted mutation of EphB1 receptor prevents development of neuropathic hyperalgesia and physical dependence on morphine in mice," Molecular Pain, vol. 4, p. 60, 2008.

[13] V. Cibert-Goton, G. Yuan, A. Battaglia et al., "Involvement of EphB1 receptors signalling in models of inflammatory and neuropathic pain," PLoS One, vol. 8, no. 1, Article ID e53673, 2013.

[14] X. Zhou, C. Zhang, C. Zhang, Y. Peng, Y. Wang, and H. Xu, "MicroRNA-182-5p regulates nerve injury-induced nociceptive hypersensitivity by targeting ephrin type-b receptor 1," Anesthesiology, vol. 126, no. 5, pp. 967-977, 2017.

[15] J. Zhao, G. Yuan, C. M. Cendan et al., "Nociceptor-expressed ephrin-B2 regulates inflammatory and neuropathic pain," Molecular Pain, vol. 6, p. 77, 2010.

[16] H. Kobayashi, T. Kitamura, M. Sekiguchi et al., "Involvement of EphB1 receptor/EphrinB2 ligand in neuropathic pain," Spine, vol. 32, no. 15, pp. 1592-1598, 2007.

[17] S. Liu, Y.-P. Liu, W. B. Song, and X.-J. Song, "EphrinB-EphB receptor signaling contributes to bone cancer pain via Tolllike receptor and proinflammatory cytokines in rat spinal cord," Pain, vol. 154, no. 12, pp. 2823-2835, 2013.

[18] S. Liu, W.-T. Liu, Y.-P. Liu et al., "Blocking EphB1 receptor forward signaling in spinal cord relieves bone cancer pain and rescues analgesic effect of morphine treatment in rodents," Cancer Research, vol. 71, no. 13, pp. 4392-4402, 2011.

[19] X.-T. Deng, M.-Z. Wu, N. Xu, P.-C. Ma, and X.-J. Song, "Activation of ephrinB-EphB receptor signalling in rat spinal cord contributes to maintenance of diabetic neuropathic pain," European Journal of Pain, vol. 21, no. 2, pp. 278-288, 2017.

[20] H.-Y. Peng, G.-D. Chen, C.-H. Lai, K.-C. Tung, J.-L. Chang, and T.-B. Lin, "Endogenous ephrinB2 mediates colon-urethra cross-organ sensitization via Src kinase-dependent tyrosine phosphorylation of NR2B," American Journal of PhysiologyRenal Physiology, vol. 298, no. 1, pp. F109-F117, 2010.

[21] M. B. Dalva, M. A. Takasu, M. Z. Lin et al., "EphB receptors interact with NMDA receptors and regulate excitatory synapse formation," Cell, vol. 103, no. 6, pp. 945-956, 2000.

[22] I. C. Grunwald, M. Korte, D. Wolfer et al., "Kinase-independent requirement of EphB2 receptors in hippocampal synaptic plasticity," Neuron, vol. 32, no. 6, pp. 1027-1040, 2001.

[23] K. Hanamura, H. R. Washburn, S. I. Sheffler-Collins et al., "Extracellular phosphorylation of a receptor tyrosine kinase controls synaptic localization of NMDA receptors and regulates pathological pain," PLoS Biology, vol. 15, no. 7, Article ID e2002457, 2017. 
[24] X.-J. Song, J.-H. Zheng, J.-L. Cao, W.-T. Liu, X.-S. Song, and Z.-J. Huang, "EphrinB-EphB receptor signaling contributes to neuropathic pain by regulating neural excitability and spinal synaptic plasticity in rats," Pain, vol. 139, no. 1, pp. 168-180, 2008.

[25] J. T. Henderson, J. Georgiou, Z. Jia et al., "The receptor tyrosine kinase EphB2 regulates NMDA-dependent synaptic function," Neuron, vol. 32, no. 6, pp. 1041-1056, 2001.

[26] A. A. Battaglia, K. Sehayek, J. Grist, S. B. McMahon, and I. Gavazzi, "EphB receptors and ephrin-B ligands regulate spinal sensory connectivity and modulate pain processing," Nature Neuroscience, vol. 6, no. 4, pp. 339-340, 2003.

[27] W. S. Xia, Y. N. Peng, L. H. Tang et al., "Spinal ephrin B/EphB signalling contributed to remifentanil-induced hyperalgesia via NMDA receptor," European Journal of Pain, vol. 18, no. 9, pp. 1231-1239, 2014.

[28] S. Slack, A. Battaglia, V. Cibert-Goton, and I. Gavazzi, "EphrinB2 induces tyrosine phosphorylation of NR2B via Srcfamily kinases during inflammatory hyperalgesia," Neuroscience, vol. 156, no. 1, pp. 175-183, 2008.

[29] L. N. Yu, L. H. Sun, M. Wang et al., "EphrinB-EphB signaling induces hyperalgesia through ERK5/CREB pathway in rats," Pain Physician, vol. 20, no. 4, pp. E563-E574, 2017.

[30] M. Yang, W. Chen, Y. Zhang, R. Yang, Y. Wang, and H. Yuan, "EphrinB/EphB signaling contributes to spinal nociceptive processing via calpain-1 and caspase-3," Molecular Medicine Reports, vol. 18, no. 1, pp. 268-278, 2018.

[31] Y. Peng, T. Zang, L. Zhou, K. Ni, and X. Zhou, "COX-2 contributed to the remifentanil-induced hyperalgesia related to ephrinB/EphB signaling," Neurological Research, vol. 41, no. 6, pp. 519-527, 2019.

[32] H.-C. Wu, C.-H. Chang, H.-Y. Peng et al., "EphrinB2 induces pelvic-urethra reflex potentiation via Src kinase-dependent tyrosine phosphorylation of NR2B," American Journal of Physiology-Renal Physiology, vol. 300, no. 2, pp. F403-F411, 2011.

[33] S. A. Theofanous, M. V. Florens, I. Appeltans et al., "EphrinB2 signaling in the spinal cord as a player in post-inflammatory and stress-induced visceral hypersensitivity," Neurogastroenterology \& Motility, vol. 32, no. 4, Article ID e13782, 2020.

[34] J. Yang, Y. Yu, H. Yu et al., "The role of brain-derived neurotrophic factor in experimental inflammation of mouse gut," European Journal of Pain, vol. 14, no. 6, pp. 574-579, 2010.

[35] Q. Li, C. Guo, M. A. Chowdhury, T. Dai, and W. Han, "TRPA1 in the spinal dorsal horn is involved in post-inflammatory visceral hypersensitivity: in vivo study using TNBS-treated rat model," Journal of Pain Research, vol. 9, pp. 1153-1160, 2016.

[36] L. Antonioli, M. Fornai, R. Colucci et al., "The blockade of adenosine deaminase ameliorates chronic experimental colitis through the recruitment of adenosine A2A and A3 receptors," Journal of Pharmacology and Experimental Therapeutics, vol. 335, no. 2, pp. 434-442, 2010.

[37] E. Lucarini, C. Parisio, J. J. V. Branca et al., "Deepening the mechanisms of visceral pain persistence: an evaluation of the gut-spinal cord relationship,” Cells, vol. 9, no. 8, 2020.

[38] E. D. Al-Chaer, M. Kawasaki, and P. J. Pasricha, "A new model of chronic visceral hypersensitivity in adult rats induced by colon irritation during postnatal development," Gastroenterology, vol. 119, no. 5, pp. 1276-1285, 2000.

[39] Y. Dong, Q.-L. Mao-Ying, J.-W. Chen, C.-J. Yang, Y.-Q. Wang, and Z.-M. Tan, "Involvement of EphB1 receptor/
ephrinB1 ligand in bone cancer pain," Neuroscience Letters, vol. 496, no. 3, pp. 163-167, 2011.

[40] R. Klein, "Bidirectional modulation of synaptic functions by Eph/ephrin signaling," Nature Neuroscience, vol. 12, no. 1, pp. 15-20, 2009. 